\title{
Some Degree-Based Topological Indices of Base-3 Sierpiński Graphs
}

\author{
Xiujun Zhang ${ }^{1,2, *}$, Hong Yang ${ }^{1,3}$, Yingying Gao ${ }^{4}$, Mohammad Reza Farahani ${ }^{5}$ \\ ${ }^{1}$ School of Information Science and Engineering, Chengdu University, Chengdu, China \\ ${ }^{2}$ Key Laboratory of Pattern Recognition and Intelligent Information Processing, Institutions of Higher Education of Sichuan Province, \\ Chengdu University, Chengdu, China \\ ${ }^{3}$ Research Institute of Big Data, Chengdu University, Chengdu, China \\ ${ }^{4}$ Colleage of Pharmacy and Biological Engineering, Chengdu University, Chengdu, China \\ ${ }^{5}$ Department of Applied Mathematics of Iran University of Science and Technology, (IUST) Narmak, Tehran, Iran
}

\section{Email address:}

woodszhang@cdu.edu.cn (Xiujun Zhang)

${ }^{*}$ Corresponding author

\section{To cite this article:}

Xiujun Zhang, Hong Yang, Yingying Gao, Mohammad Reza Farahani. Some Degree-Based Topological Indices of Base-3 Sierpiński Graphs. Science Journal of Chemistry. Vol. 5, No. 3, 2017, pp. 36-41. doi: 10.11648/j.sjc.20170503.12

Received: June 3, 2017; Accepted: July 4, 2017; Published: July 31, 2017

\begin{abstract}
In this paper, a recursive relation between base-3 Sierpiński graphs rank $\mathrm{n}$ and $\mathrm{n}-1$ of some topostructural indices is studied. Based on this relation, The formulae of the First Zagreb index, Second Zagreb index, Randić connectivity index, sum-connectivity index, Geometric-Arithmetic index and Atom-Bond Connectivity index of base-3 Sierpiński graphs are derived.
\end{abstract}

Keywords: Topological Index, Base-3 Sierpiński Graphs, Zagreb Index, Sum-Connectivity Index, Geometric-Arithmetic Index, Atom-Bond Connectivity Index, Sankruti Index

\section{Introduction}

In this paper, we only consider undirected graph without loops and multiple edges. Let $G$ be a graph, we denote $V(G)$ and $E(G)$ the vertex set and edge set of $G$, respectively. We denote by $d_{v}$ the degree of a vertex $v$ of a graph $G$. We denote by $E_{a, b}$ the set of edges $u v$ with $\left\{d_{u}, d_{v}\right\}=\{a, b\}$, i.e. $E_{a, b}=\left\{u v \mid\{a, b\}=\left\{d_{u}, d_{v}\right\}\right\}$. Let $s_{G}(v)=\sum_{u \in N(v)} d_{u}$, and the summation of the degrees of all the neighbors of a vertex $u$ be $s_{G}(u) \quad$ i.e. $s_{G}(u)=\sum_{v \in N(u)} d(v) \quad$ and $E_{a, b}^{\prime}=\left\{u v \mid\{a, b\}=\left\{s_{G}(u), s_{G}(v)\right\}\right\}$. In chemical graph theory, the vertices of the graph correspond to the atoms of molecules and the edges correspond to chemical bonds.

Klavžar et al. [9] defined the Sierpiński graphs $S(n, k)$ in 1997. The Sierpiński graphs $S(n, k)$ were related to the Tower of Hanoi problems and the universal topological spaces problems. The authors defined $S(n, k)$ as follows.

$V(S(n, k))=\{1,2,3, \cdots, k\}^{n}, k \geq 1, n \geq 1$ and two different vertices $I=\left(i_{1}, i_{2}, \cdots, i_{n}\right)$, and $J=\left(j_{1}, j_{2}, \cdots, j_{3}\right)$ are adjacent if $I \sim J$, where $I \sim J \Leftrightarrow \exists h \in\{1,2, \cdots, n\}$ such that

i) $\forall t, t<h \Rightarrow i_{t}=j_{t}$,

ii) $i_{h} \neq j_{h}$,

iii) $\forall t, t>h \Rightarrow i_{t}=j_{h} \& j_{t}=i_{h}$ [9]

Hinz et al. [5] discussed the definition and properties of Sierpiński graph, Sierpiński-type graphs, Sierpiński triangle graphs and Sierpiński-like graphs. Because we only focus on Base-3 Sierpiński Graphs $S(n, 3)$, we simplify the notation from $S(n, 3)$ to $S^{n}$. In Figure 1 the Base-3 Sierpinski Graphs are illustrated with $n \in\{1,2,3,4\}$. 


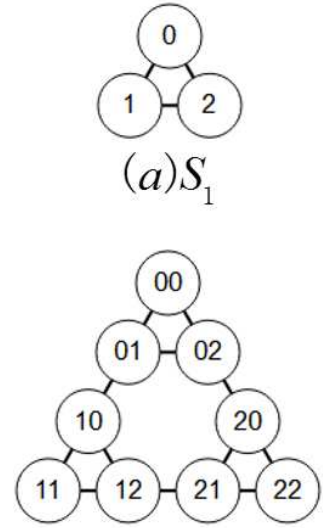

(b) $S_{2}$

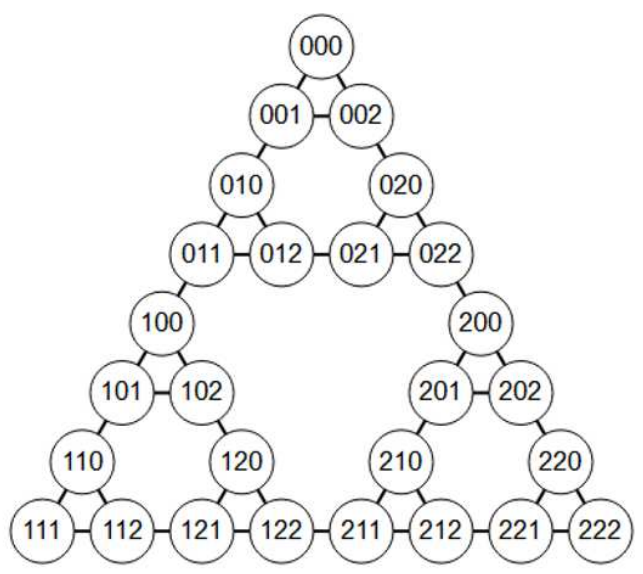

(c) $S_{3}$

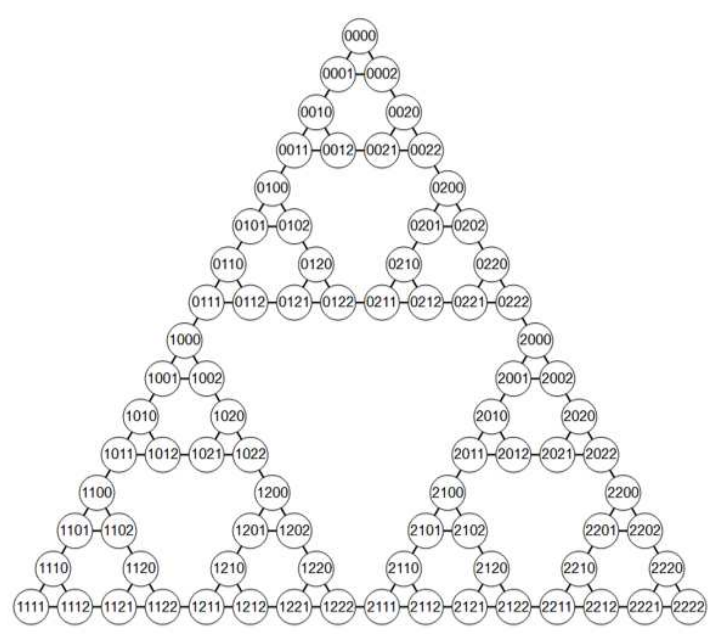

(d) $S_{4}$

Figure 1. The Sierpiński Triangles with $n=1,2,3,4$.

$S^{1}$ is $K_{3}$ and three vertices are labeled with 0,1 , and 2. $S^{2}$ consists of three copies of $S^{1}$, if we consider a $S^{1}$ as a vertex, $S^{2}$ can be considered as another type of $K_{3}$. The vertices labels of $S^{2}$ can be considered by adding $S^{1}$ vertex labels before to three copies of $S^{1}$ vertex labels. So $S^{n+1}$ is composed with three copies of $S^{n}, S^{n+1}$ is another type of $K_{3}$ that all three vertices are replaced by $S^{n}$, and $S^{n}$ vertex labels are composed by adding $S^{1}$ vertex labels before to three copies of $S^{n}$ vertex labels. The relationship of $S^{n+1}$ and $S^{n}$ is illustrated in Figure 2.

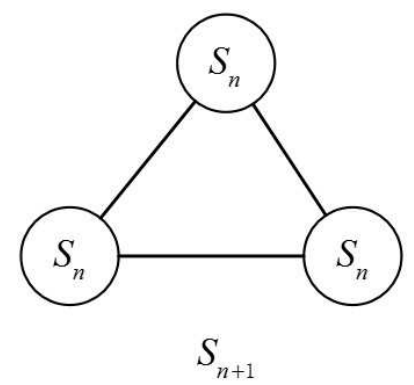

Figure 2. The Base-3 Sierpinski Graphs $S^{n+1}$.

By the definition of the Base-3 Sierpiński Graphs $\mathrm{S}^{i}$, we have

Observation 1. If $\mathrm{G}$ is an $\mathrm{S}^{\mathrm{n}}$ graph with $\mathrm{n} \geq 2$, then $E\left(S^{n}\right)=E_{2,3} \cup E_{3,3}$ with $\left|E_{2,3}\right|=6$ and $\left|E_{3,3}\right|=\frac{3^{n+1}-15}{2}$; If $\mathrm{n}=2$, then $E\left(S^{n}\right)=E_{6,8}^{\prime} \cup E_{8,8}^{\prime}$ with $\left|E_{6,8}^{\prime}\right|=\left|E_{8,8}^{\prime}\right|=6$; If $n \geq 3$, then $E\left(S^{n}\right)=E_{6,8}^{\prime} \cup E_{8,8}^{\prime} \cup E_{8,9}^{\prime} \cup E_{9,9}^{\prime}$ with $\left|E_{8,8}^{\prime}\right|=3$, $\left|E_{6,8}^{\prime}\right|=\left|E_{8,9}^{\prime}\right|=6,\left|E_{9,9}^{\prime}\right|=\frac{3^{n+1}-33}{2}$.

In chemical graph theory, the atoms of molecules can be considered as the vertices of graph and chemical bonds be considered as the edges of graph $[12,14]$. The mathematical quantities derived from a graph representation of the molecule are called graph invariants. Single indices derived from a molecular graph are called topological indices.
Topological indices are sensitive to one or more structural features of the molecule such as size, shape, symmetry, branching, and topological indices are divided into two categories: topostructural and topochemical indices [11]. In this paper, we only focus on some of the topostructural indices, including First Zagreb index $Z g_{1}(G)$, Second Zagreb index $Z g_{2}(G)$, Randić connectivity index $R(G)$, sum-connectivity index $X(G)$, Geometric-Arithmetic index $G A(G)$ and Atom-Bond Connectivity index $A B C(G)$.

The First Zagreb index were introduced by Gutman and Trinajstić [6]. When they studied the molecular structure's $\pi$ electron energy, they found the approximate expressions of M1 and M2. The First Zagreb index and the Second Zagreb index are very important topostructural indices in chemical graph theory [7].

The First Zagreb index $Z g_{1}(G)$ is defined as the sum of squares of the vertex degrees $d_{u}$ and $d_{v}$ of vertices $u$ and $v$ in $G($ see $[6,7,13])$

$$
Z g_{1}(G)=\sum_{e=u v \in E(G)}\left(d_{u}+d_{v}\right)
$$

The Second Zagreb index $Z g_{2}(G)$ is defined as the sum of squares of the vertex degrees $d_{u}$ and $d_{v}$ of vertices $u$ and $v$ in $G$ (see $[6,7,13])$ and deduced as formula (2).

$$
Z g_{2}(G)=\sum_{e=u v \in E(G)}\left(d_{u} d_{v}\right)
$$

According to the above Zagreb indices, the First Zagreb polynomial $Z g_{1}(G, x)$ and the Second Zagreb polynomial $Z g_{1}(G, x)$ have been defined. They are defined as

$$
Z g_{1}(G, x)=\sum_{e=u v \in E(G)} x^{d_{u}+d_{v}}
$$

and 


$$
Z g_{2}(G, x)=\sum_{e=u v \in E(G)} x^{d_{u} d_{v}}
$$

Kinkar and Gutman studied the properties of $Z g_{1}(G, x)$, $\mathrm{Zg}_{2}(G, x)$ for some chemical structures in 2004 [1].

The Randić index $R(G)$, proposed by Randić [10,12] in 1975 , is well correlated with a variety of physico-chemical properties of alkanes. And this index has become one of the most popular molecular descriptors. The Randić index $R(G)$ is defined on the ground of vertex degrees

$$
R(G)=\sum_{e=u v \in E(G)} \frac{1}{\sqrt{d_{u} d_{v}}}
$$

If more information about the Randić index of polymeric networks modelled by generalized Sierpiński graphs is wanted, please refer to Estradamoreno et al. [18].

The sum-connectivity index was introduced by Zhou and Trinajstić [15] which is very similar to the Randić connectivity index. The sum-connectivity index is defined (see [15]) as

$$
X(G)=\sum_{e=u v \in E(G)} \frac{1}{\sqrt{d_{u}+d_{v}}}
$$

As molecular structure descriptor based on graph theory, $X(G)$ and $\mathrm{R}(\mathrm{G})$ are both well related with each other and with the $\pi$-electronic energy of benzenoid hydrocarbons [16].

Fath-Tabar et al. were introduced the GeometricArithmetic index in 2010 (see [17]) is defined as

$$
G A(G)=\sum_{e=u v \in E(G)} \frac{2 \sqrt{d_{u} d_{v}}}{d_{u}+d_{v}}
$$

The GA index is well correlated with a variety of physicochemical properties such as entropy, enthalpy of vaporization, standard enthalpy of vaporization, enthalpy of formation and acentric factor [3,8].

By Estrada et al. [4], the Atom-Bond Connectivity index is defined as

$$
A B C(G)=\sum_{e=u v \in E(G)} \sqrt{\frac{d_{u}+d_{v}-2}{d_{u} d_{v}}}
$$

The ABC index correlates well with the experimental heats of formation of alkanes [4]. The mathematical properties of this index have been studied extensively.

The Forgotten index $F(G)$ is defined as the sum of cubic of the vertex degrees in $\mathrm{G}$ (see [13])

$$
F(G)=\sum_{v \in V(G)}\left(d(v)^{3}\right)=\sum_{u v \in E(G)}\left(d_{u}^{2}+d_{v}^{2}\right)
$$

The Harmonic index $\mathrm{H}(\mathrm{G})$ (see [19]) is defined as

$$
H(G)=\sum_{e v \in E(G)} \frac{2}{d_{u}+d_{v}}
$$

More recently, motivated by the previous research on topological descriptors and their applications, Hosamani [20] proposed the Sanskruti index which can be utilized to guess the bioactivity of chemical compounds and shows good correlation with entropy of an octane isomers.

The Sankruti index of a graph $\mathrm{G}$ is defined as

$$
\mathcal{S}(G)=\sum_{u v \in E(G)}\left(\frac{s_{G}(u) s_{G}(v)}{s_{G}(u)+s_{G}(v)-2}\right)^{3}
$$

Darafsheh [2] have discussed Wiener index formulae, Szeged index formulae and PI-index formulae of intersection graph and Hypercube (n-Cube) graph by using automorphism group method, and asserted recursion process method be good at to the graph with the same shape repeated several times, so he gave Wiener index formulae of Linear Chain, Polyphenylene and Linear Phenylene graph by using recursion formulae method. The recursion formulae method will be used to derived the formulae of the First Zagreb index $Z g_{1}(G)$, Second Zagreb index $Z g_{2}(G)$, the first Zagreb polynomial, the second Zagreb polynomial, sum-connectivity index $X(G)$, Geometric-Arithmetic index $G A(G)$, AtomBond Connectivity index $\mathrm{ABC}(\mathrm{G})$, forgotten index $\mathrm{F}(\mathrm{G})$, harmonic index $\mathrm{H}(\mathrm{G})$ and Sankruti index $\mathcal{S}(G)$ of base-3 Sierpiński graphs.

\section{Main Results}

Let $\phi(x, y)$ be a function on $\mathbb{Z} \times \mathbb{Z} \rightarrow R$ and a degree-based topological index $f(G)$ is defined as

$$
f(G)=\sum_{u v \in E(G)} \phi\left(d_{u}, d_{v}\right) .
$$

By observing the structure of the base-3 Sierpiński graphs, we have the following recursive formulation of $f\left(S^{n}\right)$ :

If $n \geq 3$, then

$$
\left\{\begin{array}{l}
f\left(S^{n}\right)=3 f\left(S^{n-1}\right)-12 \phi(2,3)+15 \phi(3,3) \\
f\left(S^{1}\right)=3 \phi(2,2) \\
f\left(S^{2}\right)=6(\phi(2,3)+\phi(3,3))
\end{array}\right.
$$

Theorem 1. $Z g_{1}\left(S^{n}\right)=3^{n+2}-15$.

Proof. It can be verified that $Z g_{2}\left(S^{1}\right)=12$ and $Z g_{2}\left(S^{2}\right)=66$ thus the theorem holds for $n=1,2$. Let $\phi(x, y)=x+y$. Then the degree-based topological index $f(G)$ in formula (1) is the first Zagreb index $Z g_{1}(G)$. By formula (1), we have If $n \geq 3$, then

$$
\left\{\begin{array}{l}
f\left(S^{n}\right)=3 f\left(S^{n-1}\right)+30 \\
f\left(S^{2}\right)=66
\end{array}\right.
$$


By solving this recursive formulation, we have $Z g_{1}\left(S^{n}\right)=$ $f\left(S^{n}\right)=3^{n+2}-15$.

Theorem 2. $Z g_{2}\left(S^{1}\right)=12$; if $n \geq 2$, we have $Z g_{2}\left(S^{n}\right)=\frac{9\left(3^{n+1}-7\right)}{2}$.

Proof. It can be verified that $Z g_{2}\left(S^{1}\right)=12$ and $Z g_{2}\left(S^{2}\right)=90$ thus the theorem holds for $n=1,2$. Now we consider the case $n \geq 3$. Let $\phi(x, y)=x y$. Then the degree-based topological index $f(G)$ in formula (2) is the second Zagreb index $Z g_{2}(G)$. By formula (2), we have if $n \geq 3$, then

$$
\left\{\begin{array}{l}
f\left(S^{n}\right)=3 f\left(S^{n-1}\right)+63 \\
f\left(S^{2}\right)=90
\end{array}\right.
$$

By solving this recursive formulation, we have $Z g_{2}\left(S^{n}\right)=\frac{9\left(3^{n+1}-7\right)}{2}$ for $n \geq 2$.

Theorem 3. $X\left(S^{1}\right)=\frac{3}{2} \quad$ if $\quad n \geq 2$, we have $X\left(S^{n}\right)=\frac{5 \sqrt{6} \times 3^{n}+24 \sqrt{5}-25 \sqrt{6}}{20}$.

Proof. It can be verified that $X\left(S^{1}\right)=\frac{3}{2}$ and $X\left(S^{2}\right)=6\left(\frac{1}{\sqrt{5}}+\frac{1}{\sqrt{6}}\right)$ thus the theorem holds for $n=1,2$. Now we consider the case $n \geq 3$. Let $\phi(x, y)=\frac{1}{\sqrt{x+y}}$. Then the degree-based topological index $f(G)$ in formula (6) is the sum-connectivity index $X(G)$. By formula (6), we have if $n$ $\geq 3$, then

$$
\left\{\begin{array}{l}
f\left(S^{n}\right)=3 f\left(S^{n-1}\right)-12 / \sqrt{5}+15 / \sqrt{6} \\
f\left(S^{2}\right)=6\left(\frac{1}{\sqrt{5}}+\frac{1}{\sqrt{6}}\right)
\end{array}\right.
$$

By solving this recursive formulation, we have $X\left(S^{n}\right)=\frac{5 \sqrt{6} \times 3^{n}+24 \sqrt{5}-25 \sqrt{6}}{20}$ for $n \geq 2$

Theorem 4. $G A\left(S^{1}\right)=3$; if $n \geq 2$, we have $G A\left(S^{n}\right)=\frac{3\left(5 \times 3^{n}-25+8 \sqrt{6}\right)}{10}$.

Proof. It can be verified that $G A\left(S^{1}\right)=3$ and $G A\left(S^{2}\right)=\frac{12 \sqrt{6}}{5}+6$ thus the theorem holds for $n=1,2$. Now we consider the case $n \geq 3$. Let $\phi(x, y)=\frac{2 \sqrt{x y}}{x+y}$. Then the degree-based topological index $f(G)$ in formula (7) is Geometric-Arithmetic index $G A(G)$. By formula (7), we have if $n \geq 3$, then

$$
\left\{\begin{array}{l}
f\left(S^{n}\right)=3 f\left(S^{n-1}\right)-\frac{24 \sqrt{6}}{5}+15, \\
f\left(S^{2}\right)=\frac{12 \sqrt{6}}{5}+6
\end{array}\right.
$$

By solving this recursive formulation, we have $G A\left(S^{n}\right)=\frac{3\left(5 \times 3^{n}-25+8 \sqrt{6}\right)}{10}$ for $n \geq 2$

Theorem 5. $A B C\left(S^{1}\right)=\frac{3}{\sqrt{2}}$, if $n \geq 2$, we have $A B C\left(S^{n}\right)=3^{n}-5+3 \sqrt{2}$.

Proof. It can be verified that $A B C\left(S^{1}\right)=\frac{3}{\sqrt{2}}$ and $A B C\left(S^{2}\right)=6\left(\frac{1}{\sqrt{2}}+\frac{2}{3}\right)$ thus the theorem holds for $n=1,2$. Now we consider the case $n \geq 3$. Let $\phi(x, y)=\sqrt{\frac{x+y-2}{x y}}$. Then the degree-based topological index $f(G)$ in formula (8) is $\mathrm{ABC}$ index $A B C(G)$. By formula (8), we have if $n \geq 3$, then

$$
\left\{\begin{array}{l}
f\left(S^{n}\right)=3 f\left(S^{n-1}\right)-12 / \sqrt{2}+10 \\
f\left(S^{2}\right)=6\left(\frac{1}{\sqrt{2}}+\frac{2}{3}\right)
\end{array}\right.
$$

By solving this recursive formulation, we have $A B C\left(S^{n}\right)=3^{n}-5+3 \sqrt{2}$ for $n \geq 2$.

Theorem 6. $F\left(S^{n}\right)=3^{n+3}-57$

Proof. It can be verified that $F\left(S^{1}\right)=24$ and $F\left(S^{2}\right)=186$ thus the theorem holds for $n=1,2$. Now we consider the case $n \geq 3$. Let $\phi(x, y)=x^{2}+y^{2}$. Then the degree-based topological index $f(G)$ in formula (9) is $F$ index $F(G)$. By formula (9), we have if $n \geq 3$, then

$$
\left\{\begin{array}{l}
f\left(S^{n}\right)=3 f\left(S^{n-1}\right)-12 \times 13+15 \times 18 \\
f\left(S^{2}\right)=186
\end{array}\right.
$$

By solving this recursive formulation, we have $F\left(S^{n}\right)=3^{n+3}-57$.

Theorem 7. $H\left(S^{1}\right)=\frac{3}{2} \quad ; \quad$ if $n \geq 2$, we have $H\left(S^{n}\right)=0.5 \times 3^{n}-0.1$

Proof. It can be verified that $H\left(S^{1}\right)=\frac{3}{2}$ and $H\left(S^{2}\right)=6\left(\frac{2}{5}+\frac{2}{6}\right)$ thus the theorem holds for $n=1,2$. Now we consider the case $n \geq 3$. Let $\phi(x, y)=\frac{2}{x+y}$. Then the degree-based topological index $f(G)$ in formula (10) is $H$ 
index $H(G)$. By formula (10), we have if $n \geq 3$, then

$$
\left\{\begin{array}{l}
f\left(S^{n}\right)=3 f\left(S^{n-1}\right)-12 \times \frac{2}{5}+15 \times \frac{2}{6} \\
f\left(S^{2}\right)=6\left(\frac{2}{5}+\frac{2}{6}\right)
\end{array}\right.
$$

By solving this recursive formulation, we have $H\left(S^{n}\right)=0.5 \times 3^{n}-0.1$ for $n \geq 2$.

Theorem 8. $\mathcal{S}\left(S^{1}\right)=\frac{512}{27} ;$ if $n \geq 2$, we have

$\mathcal{S}\left(S^{n}\right)=\frac{1448292227 \times 3^{n}-4965837723}{8429568}$

Proof. It can be verified that $\mathcal{S}\left(S^{1}\right)=\frac{512}{27}$ and $\mathcal{S}\left(S^{2}\right)=\frac{328320}{343}$ thus the theorem holds for $n=1,2$. Now we consider the case $n \geq 3$. We know that Sankruti index is defined based on $s_{G}(u)$ which is the summation of the degrees of all the neighbors of a vertex $u$, and by formula (11), we give the recursive formulation as follows which is different as formula (12).

$$
\left\{\begin{array}{l}
\mathcal{S}\left(S^{n}\right)=3 \mathcal{S}\left(S^{n-1}\right)-12 \times\left(\frac{6 \times 8}{6+8-2}\right)^{3}+15 \times\left(\frac{9 \times 9}{9+9-2}\right)^{3}, \\
\mathcal{S}\left(S^{2}\right)=\frac{328320}{343}
\end{array}\right.
$$

By solving this recursive formulation, we have $\mathcal{S}\left(S^{n}\right)=\frac{1448292227 \times 3^{n}-4965837723}{8429568}$ for $n \geq 2$.

Based on the result of Observation 1, we have

Theorem 9. If $n \geq 2$, then $Z g_{1}\left(S^{n}, x\right)=6 x^{5}+\left(\frac{3^{n+1}-15}{2}\right) x^{6}$, $Z g_{1}\left(S^{n}, x\right)=6 x^{6}+\left(\frac{3^{n+1}-15}{2}\right) x^{9}$. have

Proof. By the definition of the first Zagreb polynomial, we

$$
\begin{aligned}
Z g_{1}(G, x) & =\sum_{e=u v \in E(G)} x^{d_{u}+d_{v}} \\
& =\sum_{e \in E_{2,3}} x^{d_{u}+d_{v}}+\sum_{e \in E_{3,3}} x^{d_{u}+d_{v}} \\
& =6 x^{5}+\left(\frac{3^{n+1}-15}{2}\right) x^{6}
\end{aligned}
$$

By the definition of the second Zagreb polynomial, we have

$$
\begin{aligned}
Z g_{2}(G, x) & =\sum_{e=u v \in E(G)} x^{d_{u} d_{v}} \\
& =\sum_{e \in E_{2,3}} x^{d_{u} d_{v}}+\sum_{e \in E_{3,3}} x^{d_{u} d_{v}} \\
& =6 x^{6}+\left(\frac{3^{n+1}-15}{2}\right) x^{9} .
\end{aligned}
$$

\section{Conclusion}

In this paper, we study some of the topostructural indices of base-3 Sierpiński graphs by analysis a recursive formulation. By using this approach, we derived the formulae of the First Zagreb index $Z g_{1}(G)$, Second Zagreb index $Z g_{2}(G)$, the first Zagreb polynomial, the second Zagreb polynomial, sum-connectivity index $X(G)$, GeometricArithmetic index $G A(G)$, Atom-Bond Connectivity index $A B C(G)$, forgotten index $F(G)$, harmonic index $H(G)$ and Sankruti index $\mathcal{S}(G)$ of base-3 Sierpiński graphs.

\section{Acknowledgements}

This work was supported by Applied Basic Research (Key Project) of Sichuan Province under grant 2017JY0095, Key Project of Sichuan Provincial Department of Education under grant 17ZA0079 and Automotive Creative Design Pilot Area of Chengdu University and Longquanyi District under grant 2015-CX00-00010-ZF.

\section{References}

[1] Kinkar, C., Gutman, I. Some properties of the second Zagreb index. MATCH Commun. Math. Comput. Chem. 2004; 52; 103-112.

[2] Darafsheh, M. R. Computation of Topological Indices of Some Graphs. Acta Appl. Math. 2010; 110; 1225-1235.

[3] Vukicevic, D., Furtula, B. Topological index based on the ratios of geometrical and arithmetical means of end-vertex degrees of edges, J. Math. Chem. 2009; 46; 1369-1376.

[4] Estrada, E., Torres, L., Rodriguez, L., Gutman, I. An atom-bond connectivity index: modelling the enthalpy 128 of formation of alkanes. Indian J. Chem. Sect. 1998; 37; 849-855.

[5] Hinz, A. M., Klavzar, S., Zemljic, S. S. A survey and classification of Sierpinski-type graphs. Discrete Appl. 2017; 217; 565-600.

[6] Gutman, I., Das, K. C. The first Zagreb indices 30 years after, MATCH Commun. Math. Comput. Chem. 2004; 50; 83-92.

[7] Gutman, I., Trinajstic, N. Graph theory and molecular orbitals. Total $\pi$-electron energy of alternant hydrocarbons, Chem. Phys. Lett. 1972; 17; 535-538.

[8] Rodriguez, J. M., Sigarreta, J. M. On the GeometricArithmetic Index. MATCH Commun. Math. Comput. Chem. 2015; 74; 103-120.

[9] Klavzar, Sandi, Milutinovic, U. Graphs S (n, k), and a Variant of the Tower of Hanoi Problem. Czech. Math. J. 1997; 47; 95-104. 
[10] Randic, M. The connectivity index 25 years later. J. Mol. Graph. Model. 2001; 20; 19-35.

[11] Tomasz, P., Leszczynski, J., Mark, T. C. Molecular Descriptors, In Recent advances in QSAR studies: methods and applications; Jerzy L. Eds.; Springer Science \& Business Media: Heidelberg, Germany, 2010; pp. 39.

[12] Randic, Milan. Characterization of molecular branching. J. Am. Chem. Soc. 1975; 97; 6609-6615.

[13] Nikolc, S., Kovacevic, G., Milicevic, A., Trinajstic, N. The Zagreb indices 30 years after, Croat. Chem. Acta. 2003; 76; 113-124.

[14] Wiener, H. Correlation of Heats of Isomerization, and Differences in Heats of Vaporization of Isomers, Among the Paraffin Hydrocarbons. J. Am. Chem. Soc. 1947; 84; 26362638.

[15] Zhou, B., Trinajstic, N. On a novel connectivity index. J. Math. Chem. 2009, 46, 1252-1270.
[16] Lucic, B., Trinajstic, N., Zhou, B. Comparison between the sum-connectivity index and product-connectivity index for benzenoid hydrocarbons. Chem. Phy. Let. 2009; 475; 146148 .

[17] Fath-Tabar, G., Furtula, B., Gutman, I. A new geometricarithmetic index. J. Math. Chem. 2010; 47; 477-486.

[18] Estradamoreno, A., Rodríguezbazan, E. D., Rodríguezvelazquez, J. A. (2015). On the general randic index of polymeric networks modelled by generalized sierpiński graphs. MATCH Commun. Math. Comput. Chem. 2015; 74; 145-160.

[19] Fajtlowicz, S. On conjectures of Graffiti-II. Congr. Numer. $1987 ; 60 ; 187-197$.

[20] Hosamani, S. M. Computing Sanskruti index of certain nanostructures. J. Appl. Math. Comput. 2016; 1-9. 